\title{
A Reevaluation of the Electrophysiological Correlates of Expert Object Processing
}

\author{
Lisa S. Scott ${ }^{1}$, James W. Tanaka ${ }^{2}$, David L. Sheinberg ${ }^{3}$, \\ and Tim Curran ${ }^{1}$
}

\begin{abstract}
Subordinate-level object processing is regarded as a hallmark of perceptual expertise. However, the relative contribution of subordinate- and basic-level category experience in the acquisition of perceptual expertise has not been clearly delineated. In this study, participants learned to classify wading birds and owls at either the basic (e.g., wading bird, owl) or the subordinate (e.g., egret, snowy owl) level. After 6 days of training, behavioral results showed that subordinate-level but not basic-level training improved subordinate discrimination of trained exemplars,
\end{abstract}

\section{INTRODUCTION}

People identify objects at different levels. For example, a robin can be identified at the basic level (i.e., bird) or at the subordinate level (i.e., robin). Experts (e.g., bird or car experts) tend to identify objects within their domain of expertise at a more subordinate level relative to novices. Moreover, expert object recognition is thought to involve a categorical shift from basic to subordinate levels with training (Tanaka, 2001). However, the mechanisms underlying this categorical shift with increases in perceptual expertise are relatively unknown.

It has been suggested that the recognition of objects is accomplished through two computationally distinct systems, one for more specific, subordinate-level recognition, and another for basic-level recognition (Logothetis \& Sheinberg, 1996; Jolicoeur, 1990; Biederman, 1985; Murphy \& Brownell, 1985; Tversky \& Hemenway, 1984). This distinction is supported by research suggesting that the visual analysis of objects typically proceeds from utilizing coarse to fine visual information (e.g., Schyns \& Oliva, 1994; Kimchi, 1992). More specifically, previous research suggests that coarse shape information useful for identifying objects at the basic level occurs earlier in visual processing than does more fine, detailed information, which allows for subordinate-level recognition (Collin \& McMullen, 2005). Furthermore, the initial basic-level identification of an object has been found to depend on early processing of low spatial frequency

${ }^{1}$ University of Colorado, ${ }^{2}$ University of Victoria, ${ }^{3}$ Brown University novel exemplars, and exemplars from novel species. Eventrelated potentials indicated that both basic- and subordinatelevel training enhanced the early N170 component, but only subordinate-level training amplified the later N250 component. These results are consistent with models positing separate basic and subordinate learning mechanisms, and, contrary to perspectives attempting to explain visual expertise solely in terms of subordinate-level processing, suggest that expertise enhances neural responses of both basic and subordinate processing.
(LSF) information (Collin \& McMullen, 2005) and global shapes (Biederman \& Gerhardstein, 1993), whereas subordinate-level identification requires further visual processing of fine details (Jolicoeur, Gluck, \& Kosslyn, 1984). These findings are supported by a recent neurocomputational model of expert perceptual categorization (Nguyen \& Cottrell, 2005). This model posits the necessity of two separate systems for basic and subordinate-level processing and suggests that the brain functionally separates these levels of processing.

Based on the above findings, the timeline of visual object categorization has been hypothesized to occur from basic to subordinate levels of abstraction, defining the basic level as the "entry point" in object processing. However, in face identification, recognition of atypical exemplars, and recognition of other objects of expertise, this entry point is hypothesized to have shifted downward from the basic to the subordinate level of abstraction (Tanaka, Curran \& Sheinberg, 2005; Tanaka, 2001; Tanaka \& Taylor, 1991). Currently, the mechanisms underlying this observed shift, and how face processing as well as other expert perceptual processing differs from other types of object recognition, are contentiously debated and not well understood. For example, Grill-Spector and Kanwisher (2005) found evidence for basic-level processing preceding subordinate-level processing for both objects and faces. The current study sought to examine the electrophysiological correlates of the observed shift in entry level with increased expertise. Although it is difficult to track this shift in real-world experts, training studies, which systematically control 
subordinate and basic-level experience, allow for further elucidation of these mechanisms.

Previous investigations of real-world experts, such as car or bird experts, and training studies have established a relation between the N170 event-related potential (ERP) component and expert object recognition (Busey \& Vanderkolk, 2005; Gauthier, Curran, Curby, \& Collins, 2003; Rossion, Gauthier, Goffaux, Tarr, \& Crommelinck, 2002; Tanaka \& Curran, 2001). For example, Tanaka and Curran (2001) report enhanced N170 amplitude when bird experts view birds compared to dogs and when dog experts view dogs compared to birds. This N170 difference is also seen in car experts (Gauthier et al., 2003). In addition, fingerprint experts show an inversion effect while viewing fingerprints, relative to novices (Busey \& Vanderkolk, 2005). Studies in which participants were trained to better differentiate novel objects have also found N170 changes over training. For example, participants who were trained with families of novel visual shapes called "blobs" exhibit enhanced N170 amplitude to these blobs (Curran, Tanaka, \& Weiskopf, 2002) and participants trained with novel objects called "greebles" show N170 inversion effects, similar to what is seen with faces (Rossion, Gauthier, et al., 2002). Combined, the above results suggest that the N170 is modulated by perceptual expertise; however, it is unclear whether increased expertise at the basic or the subordinate level (or both) accounts for these electrophysiological differences. Moreover, it is currently unclear how the amount of category experience, as opposed to basicand subordinate-level learning, modulates the N170.

The N250 is an ERP component that is associated with face processing (Schweinberger, Pickering, Burton, \& Kaufmann, 2002). This component is sensitive to repetition and familiarity and appears to be related to the recognition of individual people (Tanaka, Curran, Porterfield, \& Collins, 2006; Itier \& Taylor, 2004a; Schweinberger, Huddy, \& Burton, 2004). Furthermore, a recent investigation using source localization postulates that the N250 (and not the N170) may originate from the fusiform gyrus, an area in the occipital-temporal cortex associated with face processing (Schweinberger et al., 2004). Given previous arguments that the FFA is generally important for subordinate-level processing (e.g., Gauthier et al., 2002), the N250 might also be related to subordinatelevel expertise. However, the specificity of this component has not yet been investigated using nonface objects of expertise.

The present study incorporates behavioral and electrophysiological methods to investigate the acquisition of perceptual expertise. Similar to Tanaka et al. (2005), adult participants learned to classify 10 species of wading birds and 10 species of owls at either the subordinate (species, e.g., snowy owl) or basic (wading bird) level of abstraction (see Figure 1). Training included 6 days of perceptual learning tasks with the amount of learning trials equated for both the basic- and subordinate-level training. Pre- and posttraining performance was measured by using a serially presented same/different matching task while ERPs were recorded. This task tested subordinate knowledge for species trained at the subordinate level versus those trained only at the basic level. Replicating previously reported findings (Tanaka et al., 2005), we found that perceptual discrimination transferred to new exemplars and new species for birds learned at the subordinate level but not at the basic level. Electrophysiological results revealed that relative to pretraining levels, an enhanced N170 was elicited by birds from both the owl and wading bird families - regardless of whether they were trained at the basic or subordinate level. These findings suggest that the previously reported expert N170 might be attributable to the greater basic-level category experience that experts have to objects in their domain of expertise relative to novices. In contrast, only those birds that were learned at the subordinate level elicited an enhanced N250, and this effect generalized to new exemplars and novel species of subordinate-level categories. Source analyses of these components revealed distinct dipole locations for the N170 and N250, suggesting that different neural generators might mediate these potentials. Together, these findings provide electrophysiological evidence for distinct mechanisms modulating basic- and subordinate-level object recognition. Furthermore, based on the results of the current investigation, the acquisition of expertise appears to be associated with (1) increased basic-level category familiarity, including coarse category shape and structure information and (2) increased subordinate-level processing of fine details related to within-category individuation. Furthermore, these results do not support previous N170 reports suggesting that the N170 is specifically related to expert perceptual processing at the subordinate level.

\section{METHODS \\ Participants}

Participants included 16 right-handed undergraduates recruited from the University of Colorado at Boulder (6 women). Seven participants completed subordinatelevel training with wading birds and basic-level training with owls, and 9 completed subordinate-level training with owls and basic-level training with wading birds. One participant was excluded from the final sample because of poor behavioral performance. All participants gave informed consent to participate in this study.

Each participant participated in eight sessions on different days within a 2-week period. ERPs were recorded on the first and last sessions. Subjects were paid $\$ 15$ per hour for ERP sessions, $\$ 10$ per hour for behavioral training sessions, and were paid a bonus of $\$ 20$ for completing all eight sessions within a 2-week period. 
Figure 1. Examples of stimuli. The top pictures are examples of trained exemplars of owls (Species A) and wading birds (Species B). Training level (basic vs. subordinate) for owls and wading birds was counterbalanced across subjects. The middle pictures are examples of owls (Species A) and wading birds (Species B) in the trained species/untrained exemplars condition. The bottom pictures are examples of owls (Species C) and wading birds (Species D) in the untrained species condition.

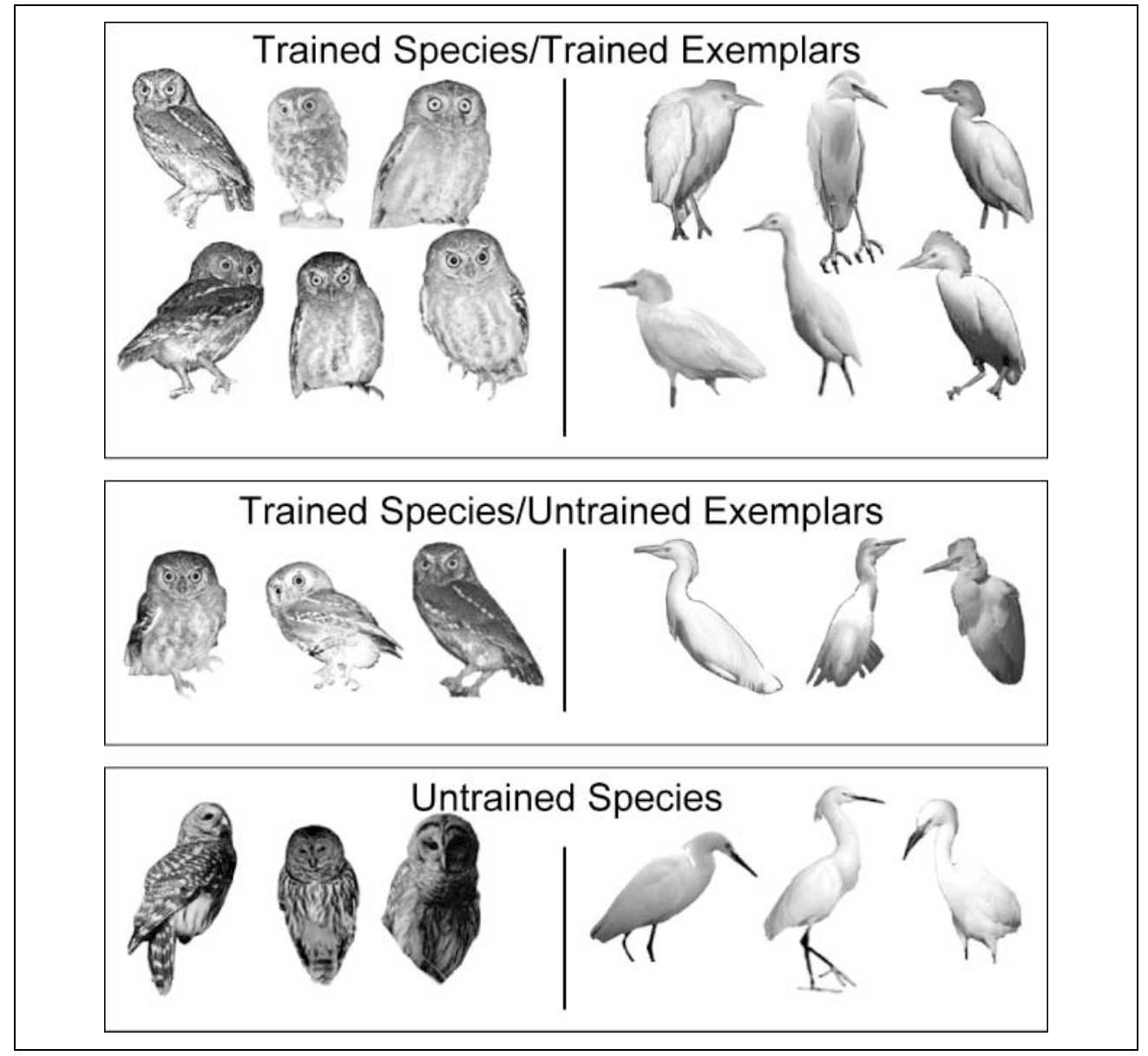

\section{Stimuli and Apparatus}

Stimuli were digitized photographs of owls and wading birds obtained from bird identification field guides or ornithological Web sites. The training set was composed of six exemplars of 10 different species from the owl family (barn owl, barred owl, boreal owl, burrowing owl, eastern screech owl, elf owl, Eurasian eagle owl, flammulated owl, and the great gray owl) and 10 species from the wading bird family (American bittern, blackcrowned night heron, cattle egret, glassy ibis, great blue heron, great egret, green heron, least bittern, limpkin, and the little blue heron). The test set of stimuli included the trained exemplars, untrained exemplars of trained species, and untrained species. The untrained exemplars included new instances of new species of owls (long-eared owl, northern hawk owl, northern pygmy owl, northern saw-whet owl, short eared owl, snowy owl, spotted owl, and the whiskered screech owl) and wading birds (reddish egret, sandhill crane, snowy egret, tricolored heron, white ibis, whooping crane, wood stork, and the yellow-crowned night heron) not learned in training (see Figure 1). The images were cropped to show only the bird and were placed on a white background. Stimuli were displayed on a 15 -in. Mitsubishi flat-panel monitor.

\section{Procedure}

All procedures were approved by the Institutional Review Board at the University of Colorado and were conducted in accordance with this approval.

\section{Electrophysiological Pre- and Posttraining Assessment}

Before and after training, participants completed a subordinate-level sequential matching task that has previously been shown to be sensitive to differences in levels of perceptual expertise (Tanaka et al., 2005; Gauthier et al., 2003) while an electroencephalogram (EEG) was recorded. Participants were shown a stimulus for $800 \mathrm{msec}$ followed by a fixation point for 800 $1000 \mathrm{msec}$ and then another image for an additional 800 msec. Then the participants were immediately presented with a question mark and were required to indicate whether the two images were of the SAME (e.g., two screech owls) or of a DIFFERENT (e.g., snowy owl and screech owl) species. The question mark remained on the screen until a response was made. SAME trials were always different exemplars of the same species. DIFFERENT trials included two exemplars of different species within the same family. Different trial lures were selected randomly (without replacement) from a 
pool of all other species within the same family. Thus, a subordinate-level discrimination was required for species trained at the subordinate and at the basic level. This task consisted of 368 trials. Half of the trials were wading birds and half owls. To monitor changes related to training, the same stimuli were included in the pre- and posttest task. All stimuli were randomly ordered. Across both same and different trials, there were three different types of trials: (1) trained species/ trained exemplars, (2) trained species/untrained exemplars, and (3) untrained species/untrained exemplars. The trained species/trained exemplars condition included 60 images from the training sessions, the trained species/new exemplars included 60 new pictures of the trained species, and finally the untrained species/untrained exemplars included 64 images of new species that were never trained. Trial numbers across conditions were not equated because of the limited number of species types available.

\section{Behavioral Training Tasks}

After the pretraining assessment, participants completed 6 days of training. One group completed subordinatelevel training with wading birds and basic-level training with owls and the other group did the opposite. The first training task was a naming task, the second task a category verification task, and the third a matching task.

Training task 1 (naming). Participants learned to label different species of either owls or wading birds. Participants completed six blocks of training on the first day and nine blocks of training on Days 2-6. Participants started this task with two species of each family (i.e., two wading birds and two owls) and increased by one more species in each family every time they got a block of trials correct. On Day 1, seven species within each family were trained. On Days 2-6, all 10 species within each family were trained. Within each block, three exemplars of each species were presented. The exemplars rotated across blocks and days, and all trained exemplars were presented on each day. The first presentation of each species was labeled, for example, a screech owl with either the subordinate-level label "This is Species Y" or the basic-level label "This is Other." Arbitrary labels, rather than actual family/species names (e.g., owl, screech owl) were used to help reduce the effects of prior knowledge. For the subordinate-level training participants then pressed the "Y" key whenever they saw a screech owl. For the basic-level training participants pressed the "O" key whenever they saw an owl. The label was only present for the first presentation of each species in each block. Participants were presented with an equal number of basic and subordinate trials. Participants were required to score $100 \%$ in each block to move on; otherwise, the block was restarted. Feedback, including the correct answer, was given for $1500 \mathrm{msec}$ for incorrect responses.

Training task 2 (category verification). Participants were presented with a subordinate-level or basic-level bird label, for example, "Species Y" (subordinate) or "Other Family" (basic) for $500 \mathrm{msec}$, followed by a fixation cross for $250 \mathrm{msec}$ and a picture of a bird for $500 \mathrm{msec}$. If the image and the label matched, the participant pressed a key for SAME. If they did not match, they pressed a key for DIFFERENT. SAME trials included an exemplar from the same family (basic) or same species (subordinate). DIFFERENT trials included an exemplar from a different family (basic) or a different species within the same family (subordinate). On the first day of training there were 48 trials presented: 2 (subordinate/basic) $\times 2$ (same/different) $\times 12$ (exemplars). Lures for the DIFFERENT trials were selected from the previously trained exemplars. Thus, on Day 1, three exemplars from the six subordinate trained species were presented twice for a total of 36 trials (12 subordinate-level SAME trials, 12 subordinate-level lures, and 12 basic-level lures) and one exemplar from each of the basic trained species were presented twice for a total of 12 trials (all 12 trials were basic-level SAME trials). On Days 2-6, three exemplars from the 10 subordinate trained species were presented one time for a total of 30 trials (10 subordinate-level SAME trials; 10 subordinate-level lures; and 10 basic-level lures) and one exemplar from the species trained at the basic level was presented one time for a total of 10 trials (all 10 trials were basic-level SAME trials). Exemplars were rotated across days so each exemplar was presented between five and six times throughout the training. Participants were given correct or incorrect feedback for $500 \mathrm{msec}$ following the response.

On the first day of training, participants completed this task once, but on all subsequent training days they also completed a speeded version of this task. In this version, participants were given $1 \mathrm{sec}$ to respond or the trial was marked incorrect. The same number of trials/ conditions was used in the speeded version of this task.

Training task 3 (matching). Participants were presented with a word label, for example, "This is Species Y" or "This is Other," for $500 \mathrm{msec}$ followed by a presentation of two side-by-side pictures of birds. Participants then pressed one key if the correct or matching stimulus was on the left and another if it was on the right. The paired stimuli remained on the screen until the participant responded. On the first day of training there were 48 trials presented: 2 (subordinate/basic) $\times 2$ (left/right) $\times 12$ (exemplars). For subordinate trials, targets were paired with a subordinate trained lure. For basic trials, targets were also paired with a subordinate trained lure. Within the subordinate-level trials, two exemplars of each of the six subordinate-training species were presented four times, twice as targets and 
twice as lures. Within the basic-level trials, two exemplars of the six basic-training species were presented twice as targets. On Days 2-6, this task included 40 trials (20 basic and 20 subordinate). Within the subordinatelevel trials, two exemplars of each of the 10 trained species were presented twice (once as a target and once as a lure). Within the basic-level trials, two exemplars of the 10 species, trained at the basic level, were paired with subordinate-level lures and presented once as targets. Participants were given correct and incorrect feedback for 500 msec following the response.

\section{Electrophysiological Methods}

Scalp voltages were collected with a 128-channel Geodesic Sensor Net (Tucker, 1993) connected to an ACcoupled, 128-channel, high-input impedance amplifier (200 M $\Omega$, Net Amps, Electrical Geodesics Inc., Eugene, OR). Amplified analog voltages (0.1-100 Hz band pass) were digitized at $250 \mathrm{~Hz}$ and collected continuously. Individual sensors were adjusted until impedances were less than $40 \mathrm{k} \Omega$. Trials were discarded from analyses if they contained eye movements (vertical electrooculogram channel differences greater than $70 \mu \mathrm{V}$ ) or more than 10 bad channels (changing more than $100 \mu \mathrm{V}$ between samples, or reaching amplitudes over $200 \mu \mathrm{V}$ ). EEG from individual channels that was consistently bad for a given participant was replaced using a spherical interpolation algorithm (Srinivasan, Nunez, Silberstein, Tucker, \& Cadusch, 1996).

Stimulus-locked ERPs were baseline-corrected with respect to a 100-msec prestimulus recording interval and digitally low-pass filtered at $40 \mathrm{~Hz}$. An average-reference transformation was used to minimize the effects of reference-site activity and accurately estimate the scalp topography of the measured electrical fields. Because of low trial counts, ERPs included trials in which the subject responded either "same" or "different," and all correct and incorrect trials. Collapsed across these factors and conditions, there was an average of 58.0 (range, 48-64; $S D=3.4$ ) trials per subject within each condition for birds trained at the subordinate level and 57.2 (range, 45-64; $S D=3.9$ ) trials within each condition for the birds trained at the basic level.

\section{Dipole Source Analysis}

Dipole source analyses were conducted using by Brain Electromagnetic Source Analysis (BESA, Version 5.0). Modeling in the present experiment used the four-shell spherical head model, which accounts for differing conductances of the brain, bone, cerebrospinal fluid, and scalp. Symmetry constraints with respect to location were used for dipole pairs. No other localization constraints were used. Localization coordinates are reported according to the Talairach and Tournoux (1988) atlas.

\section{Statistical Procedure}

\section{Statistical Analysis of Behavioral Measures}

To determine whether there was behavioral evidence of an entry-level shift from basic to subordinate processing across training, measures of reaction time (RT) for the category verification and matching tasks were entered into a $2 \times 6$ multivariate ANOVA (MANOVA) with two levels of category level (basic, subordinate) and six levels of training day (Day 1 , Day $2, \ldots$, day 6 ). In addition, $d^{\prime}$ analyses were conducted on the pre- and postsequential matching task to determine changes in discriminability after training. Measures of $d^{\prime}$ were entered into a $2 \times 2 \times$ 3 including two levels of test (pretest, posttest), two levels of categorization (basic, subordinate), and three levels of condition (training species/trained exemplars, trained species/new exemplars, and untrained species).

\section{Statistical Analysis of Electrophysiological Measures}

Electrophysiological analyses of each individual component of interest (N170; N250) was analyzed using separate $2 \times 2 \times 3 \times 2 \times 2$ MANOVAs including two levels of test (pretest, posttest), two levels of categorization (basic, subordinate), three levels of condition (trained species/trained exemplars, trained species/new exemplars, and untrained species), two levels of stimulus presentation (first/second within a trial), and two hemispheres (right, left).

Mean N170 amplitude was calculated from 147 to $211 \mathrm{msec}$ poststimulus onset (mean latency [179 msec] \pm 2SD [15.89 msec]). The channels were selected by identifying the electrode locations in the right and left hemisphere with the largest N170 across all conditions (channels 59 and 92, between standard locations T5/T6 and P3/P4). Analyses were conducted on averaged ERPs across these channels and the six immediately adjacent channels within each hemisphere (see Figure 4).

Mean N250 amplitude was analyzed across conditions from 230 to 330 msec poststimulus onset. This window was selected for analyses based on previous reports of the N250 (Schweinberger et al., 2002, 2004). Based on previous research (Tanaka et al., 2006), the same electrode groups and analyses used for the N170 were used for the N250.

\section{RESULTS}

\section{Behavioral Results}

During training tasks, RT measures were used to monitor the effects of training. RTs were computed for correct responses only. The category verification and matching tasks all showed significant interactions between training day and category level such that subordinate-level performance became increasingly similar to basic-level performance across days: verification, $F(5,10)=7.66$, $p=.003$; speeded verification, $F(4,11)=3.42, p=.05$; 
matching, $F(5,11)=12.88, p=.0001$ (see Figure 2). However, on the last day of training, RT to basic-level trials was still significantly faster than RT to subordinatelevel trials across all tasks $(p<.001)$. Accuracy for all tasks across all 6 days was at or near ceiling. Because of large variability between the number of completed blocks across subjects, analyses were not conducted for the naming task.

Subordinate-level discrimination performance was assessed before and after training across trained and untrained exemplars and species by using a successive matching task. Subordinate-level discriminations of
Figure 2. Reaction time changes across training. Behavioral data illustrating an entry-level shift in reaction time (RT) across the three different training tasks: (A) verification, (B) matching, and $(\mathrm{C})$ speeded verification. Error bars are standard error of the mean.

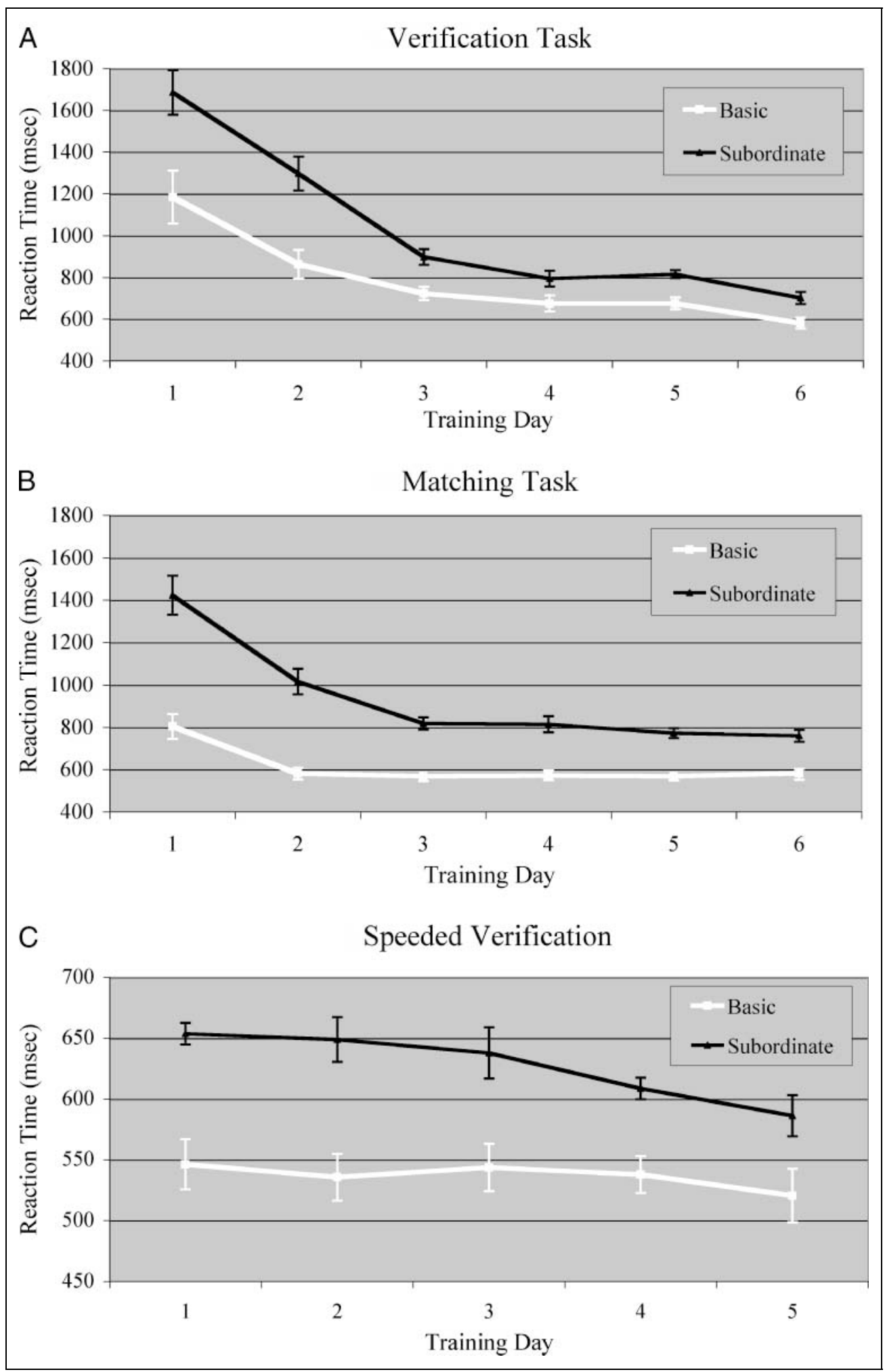


birds trained at both the subordinate and the basic level were tested in this task. Basic-level discriminations were not tested. Pre- and postmatching task performance, measured with $d^{\prime}$, benefited only from subordinatelevel training, and these benefits generalized from trained exemplars to untrained exemplars and untrained species (see Figure 3). Training level (basic, subordinate) significantly interacted with pre/post, $F(1,15)=$ 267.21, $p=.0001$. When each training condition was considered separately, $d^{\prime}$ significantly increased from pre- to posttraining at the subordinate level, $F(1,15)=$ 266.14, $p=.0001$, but basic-level training had no effects, $F(1,15)=.364, p=.555$. Planned comparisons revealed that subordinate-level training increased $d^{\prime}$ for all conditions: trained exemplars, $t(15)=-6.70, p=$ .001 ; untrained exemplars of trained species, $t(15)=$ $-6.81, p=.001$; and untrained species, $t(15)=-3.94$, $p=.01$.

\section{Electrophysiological Results}

\section{N170}

Both subordinate- and basic-level training increased N170 amplitude in a manner that generalized across all conditions. Overall, there was a significantly greater N170 posttraining compared to pretraining, $F(1,15)=$ $19.13 p=.001$ (see Figures 4 and $5 \mathrm{~A}$ ), but the training effect did not interact with categorization level, $F(1,15)=.043, p=.839$ (see Figure 6 for topographic maps). $t$ tests confirmed that these training effects were significant for all conditions (trained exemplars, un- trained exemplars, untrained species) following either subordinate- or basic-level training (all $p s<.01$ ). Analyses also revealed significant N170 differences between trained species/trained exemplars, trained species/untrained exemplars, and untrained species, $F(2,14)=$ $4.84, p=.025$. Further analyses of this effect revealed a decrease in magnitude from trained species/trained exemplars to trained species/untrained exemplars to untrained species (pairwise comparison of ordered means, $p$ s range from .05 to .07).

\section{N250}

Only subordinate-level training, but not basic-level training, increased N250 amplitude, and these subordinate training effects generalized across all conditions. Overall there was a significantly greater N250 for birds trained at the subordinate versus the basic level, $F(1,15)=5.61$, $p=.032$. This main effect is qualified by a significant interaction between pre- and posttraining and basic versus subordinate training, $F(1,15)=36.67, p=.0001$, and between pre- and posttest, basic versus subordinate training and hemisphere, $F(1,15)=5.55, p=.032$. These interactions reveal a larger posttest N250 for birds trained at the subordinate level in the right hemisphere (see Figures $4,5 \mathrm{~B}$, and 6). $t$ tests indicated that these right-hemisphere subordinate-level training effects were significant for all conditions (trained exemplars, untrained exemplars, untrained species, all $p s<.05)$.

There were also significant differences between the first and second stimulus presented within a trial. The second presentation of a stimulus elicited a smaller N250
Figure 3. Pre- and posttraining matching performance. Behavioral $d^{\prime}$ scores (mean and standard errors) across all conditions for the preand posttraining sequential matching task.

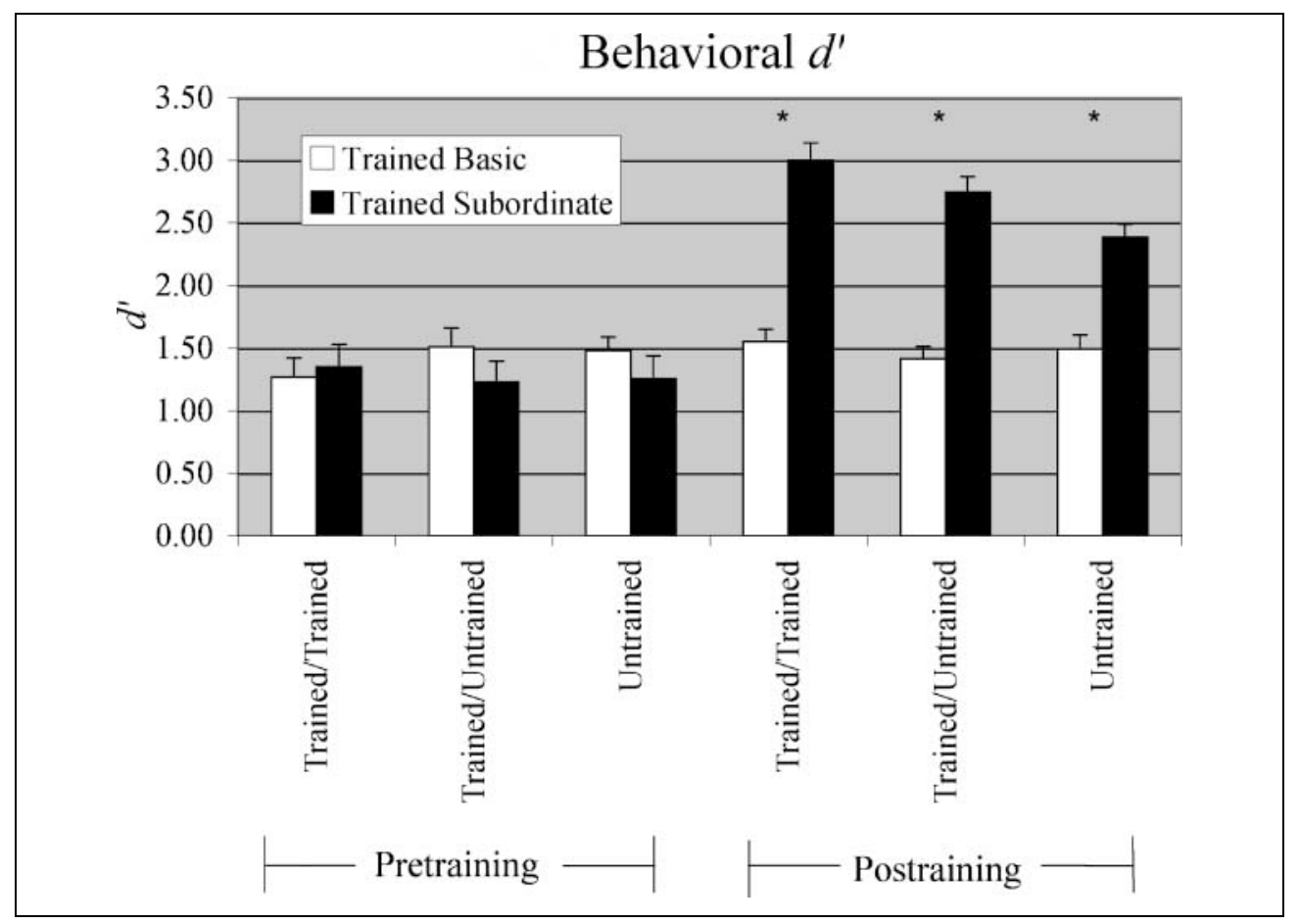


Figure 4. ERP waveforms. The left graph represents an average of electrodes in the left hemisphere $(59,51,52,58$, 60,65 , and 66) and the right graph represents an average of electrodes in the right hemisphere $(92,91,93,97$, 98,85 , and 86).

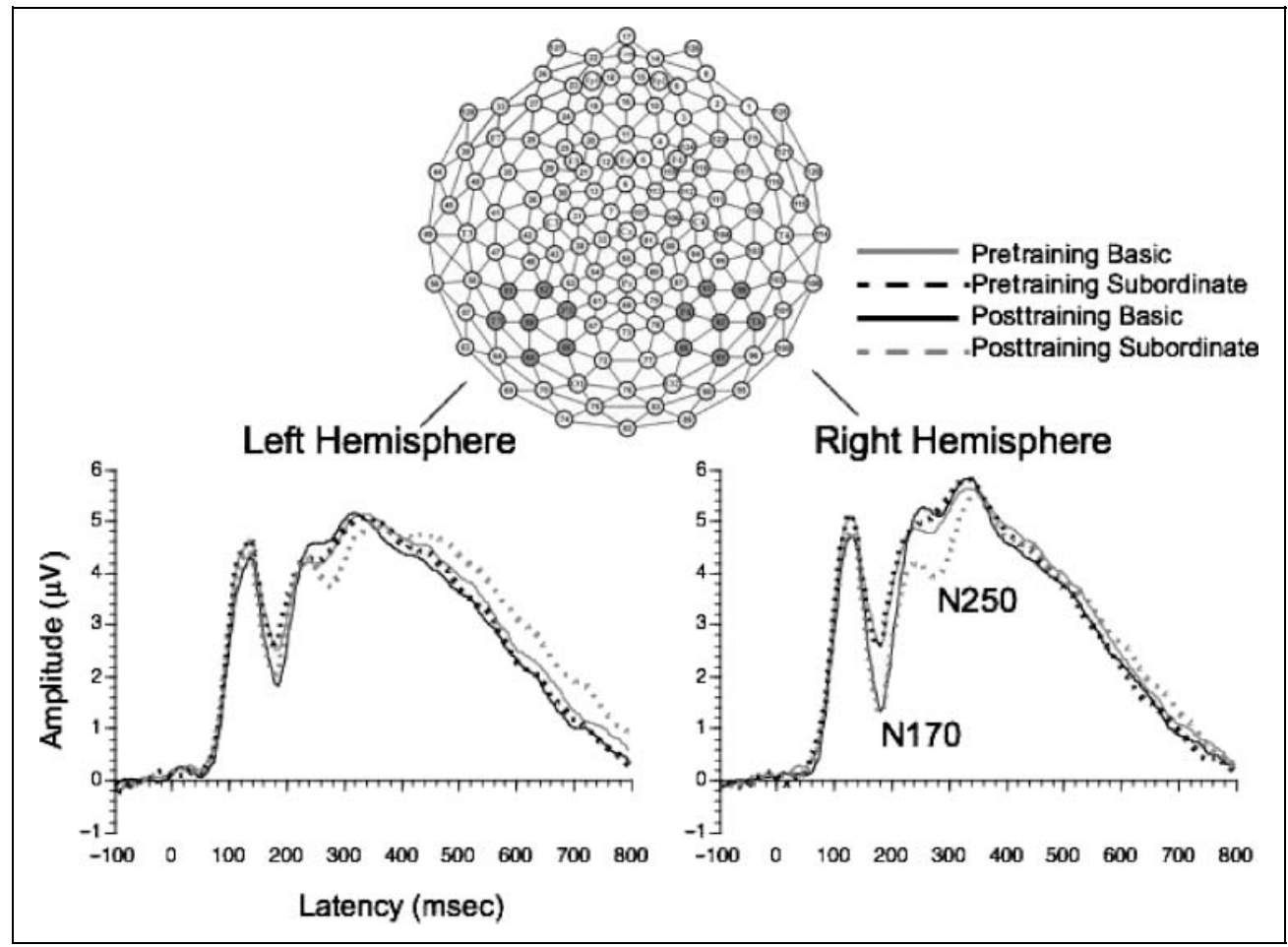

than the first presentation of a stimulus, $F(1,15)=9.85$, $p=.007$. This presentation-order effect was greater in the right hemisphere compared to the left hemisphere, $F(1,15)=4.38, p=.05$. Moreover, the difference between the first and second presentation interacted with subordinate/basic training, $F(1,15)=6.02, p=.03$. This interaction was due to a smaller (less negative) mean amplitude N250 for the second stimulus presentation $(M=5.14 \mu \mathrm{V}, S D=.59)$ compared to the first stimulus presentation $(M=4.21 \mu \mathrm{V}, S D=.52)$ for subordinate, but not basic-level trials (first presentation $M=4.8 \mu \mathrm{V}, S D=.43$; second presentation $M=5.3 \mu \mathrm{V}$, $S D=.552)$. This interaction did not further interact with pre/post, so did not modulate the training effects of primary interest.

Visual inspection of the topography of these effects (see Figure 6) indicated additional, more lateral electrode locations exhibiting an N250. Thus, in addition to the above analyses, more lateral electrode locations were also analyzed (right hemisphere: 101, 102, 108; left hemisphere: $57,58,63$ ) in a separate MANOVA to identify possible topographic differences between the N170 and the N250. Analyses of these regions replicated the interaction between pre- and posttest and subordinate- versus basic-level training, $F(1,15)=5.57, p=.032$, revealing a greater N250 for birds trained at the subordinate versus the basic level at posttest.

\section{Dipole Source Analysis}

Source localization was performed for the N170 and N250 by using BESA (Version 5.0). Source analyses were conducted for the posttest subordinate condition in which both components were most discernable. The N170 and N250 were localized by using a component onset-to-peak window $(\mathrm{N} 170=148-184 \mathrm{msec}$; $\mathrm{N} 250=$ 232-280 msec; Scherg \& Berg, 1996). Spatial principle components analysis (PCA) revealed one factor for the N170 (97\% of the variance explained) and one factor for the N250 (99.8\% of the variance explained); therefore, we fitted one pair of laterally symmetric sources for each component (see Figure 7). The Talairach coordinates for the centers of activity were $x= \pm 8.0, y=-58.2, z=4.0$ (residual variance $[\mathrm{RV}]=3.3 \%$ ) for the $\mathrm{N} 170$ and $x=$ $\pm 41.6, y=-62.1, z=-0.9(\mathrm{RV}=2.7 \%)$ for the $\mathrm{N} 250$. The center of activity for the N170 is in the lingual gyrus of the occipital lobe and the center of activity for the N250 is between the subgyral and inferior temporal gyral regions of the posterior temporal lobe.

\section{DISCUSSION}

The current investigation was designed to further investigate the mechanisms associated with acquisition of perceptual expertise using both behavioral and electrophysiological measures. Behavioral results from the present investigation replicate previous findings (Tanaka et al., 2005) indicating a decrease in RT to subordinatelevel discriminations, which approaches the RT to basiclevel discriminations. These data further support the presence of an entry-level shift in processing from the basic to subordinate level with expertise training. The results of the posttraining sequential matching task suggest that training enhanced subordinate-level 
Figure 5. Amplitude changes across training. Bar graphs of the mean and standard error of the average amplitude across conditions in the pre- and posttests across hemispheres for the N170 (A) and in the right hemisphere for the N250 (B).

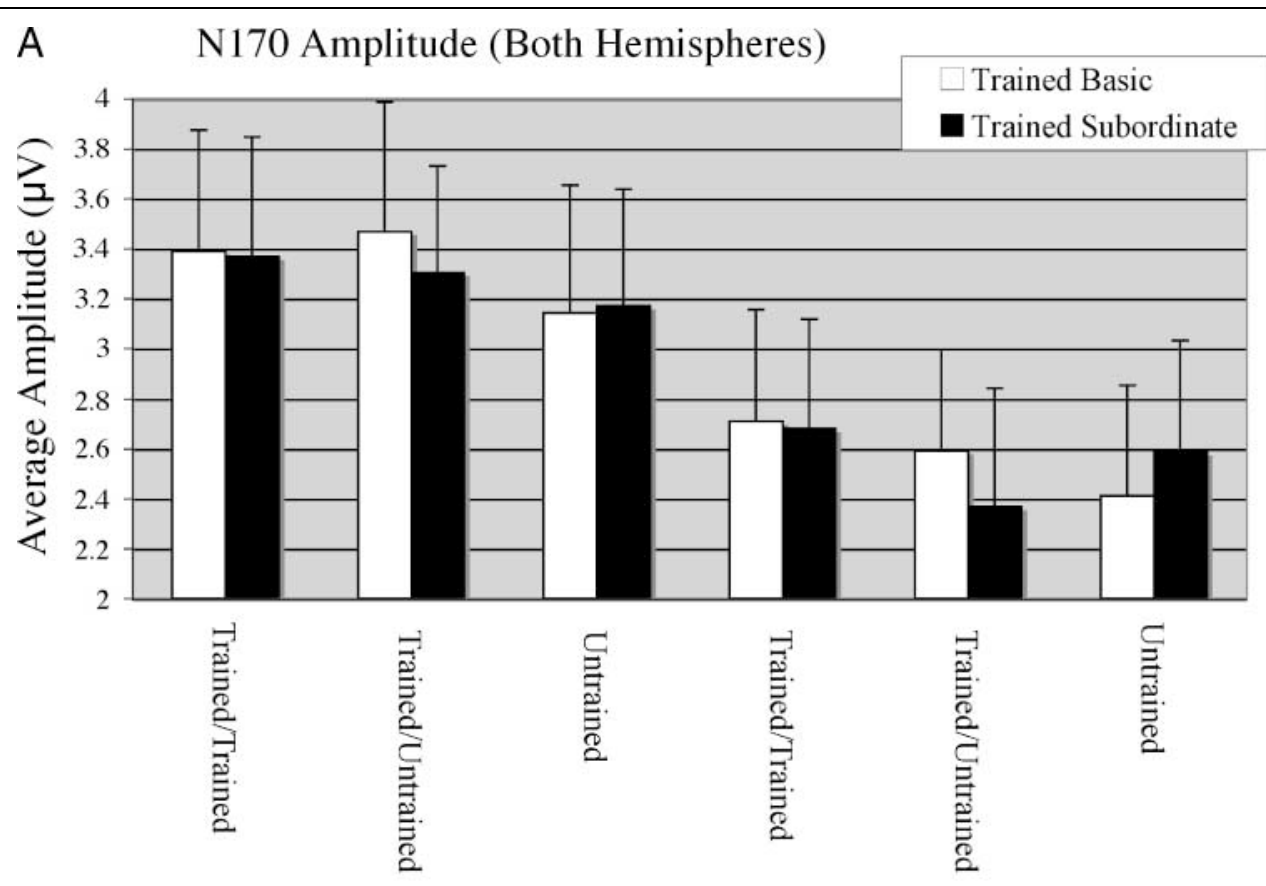

B N250 Amplitude (Right Hemispheres)
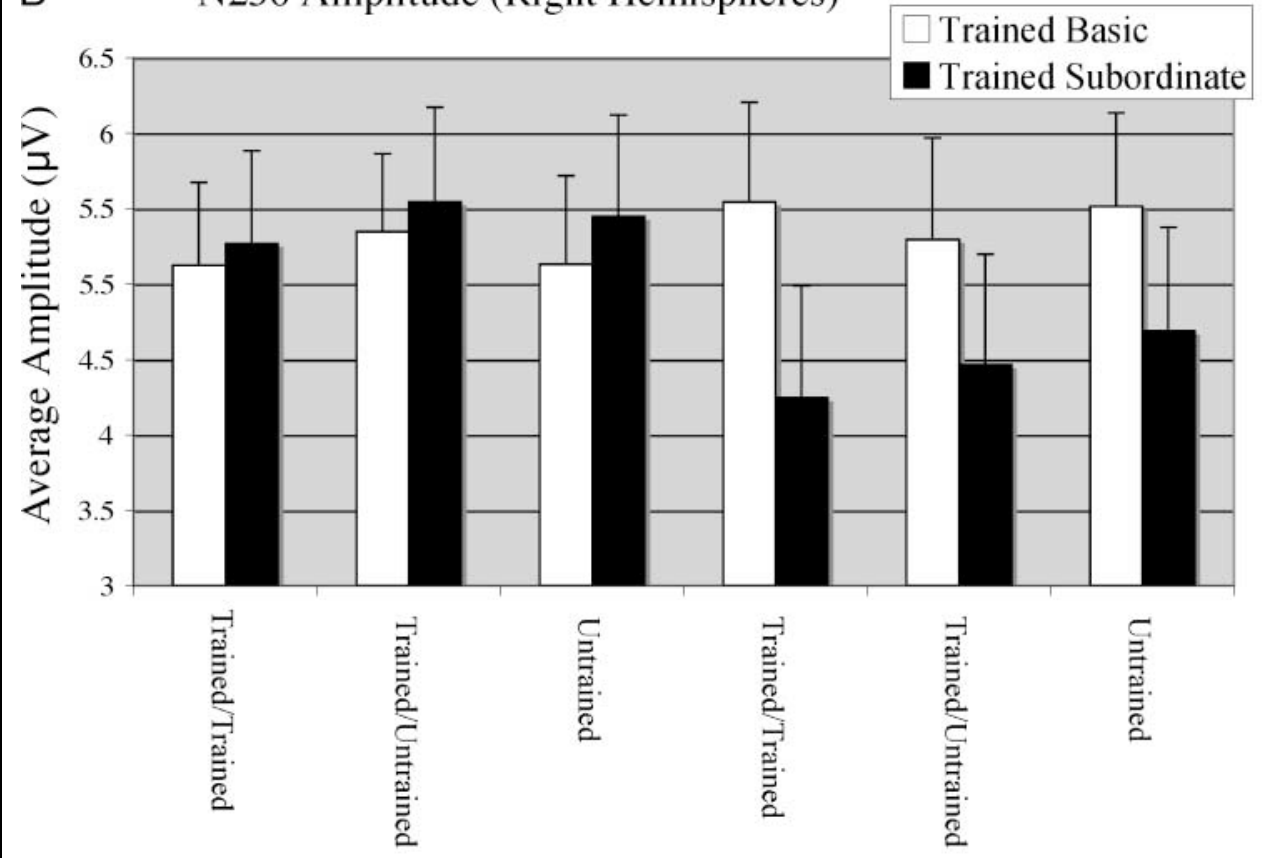

莺

Postraining discrimination of exemplars trained at the subordinate but not the basic level, and that this enhanced discrimination generalized to novel exemplars of trained species and novel exemplars of untrained species within the owl or wading bird family but not across bird families. These data suggest that subordinate-level training in the present experiment led to increases in perceptual discrimination associated with category learning.
At the electrophysiological level, basic- and subordinate-level training also produced changes in the N170 and N250 brain potentials. Based on previous reports of the relation between the N170 and perceptual expertise (Gauthier et al., 2003; Rossion, Curran, \& Gauthier, 2002; Rossion, Gauthier, et al., 2002; Tanaka \& Curran, 2001), we expected to see increases in N170 amplitude associated with only subordinate-level bird training. 
Figure 6. Topographic maps of the difference between the pre- and posttraining amplitudes for the N170 (left) and the N250 (right).

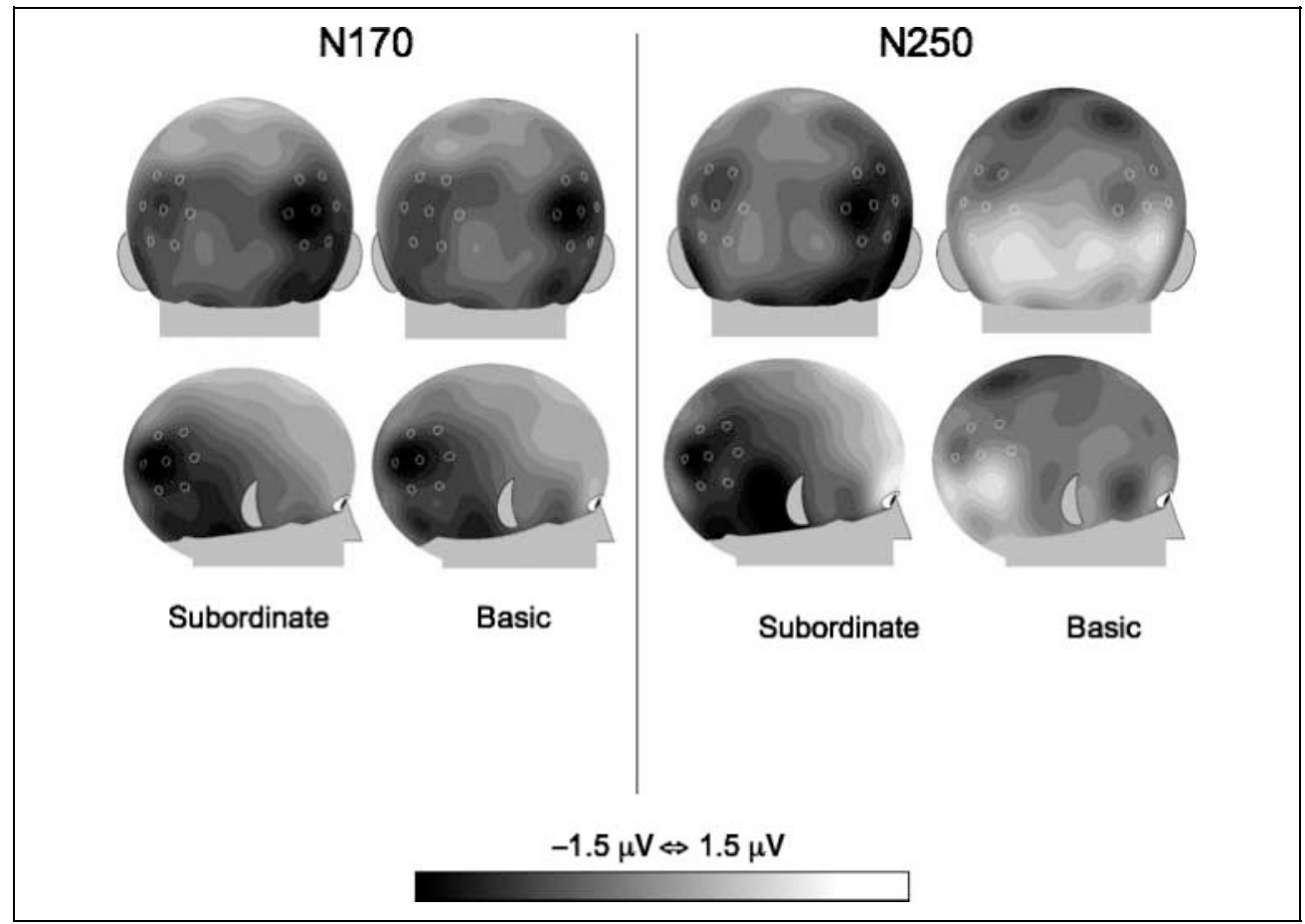

However, counter to this expectation, participants showed an enhanced N170 to owls and wading birds regardless of whether they were trained at the subordinate or the basic level. These findings suggest that more general effects of basic-level category experience mediate N170 amplitude. Based on this result, the previously reported N170 differences between real-world experts and novices might reflect the expert's greater experi-

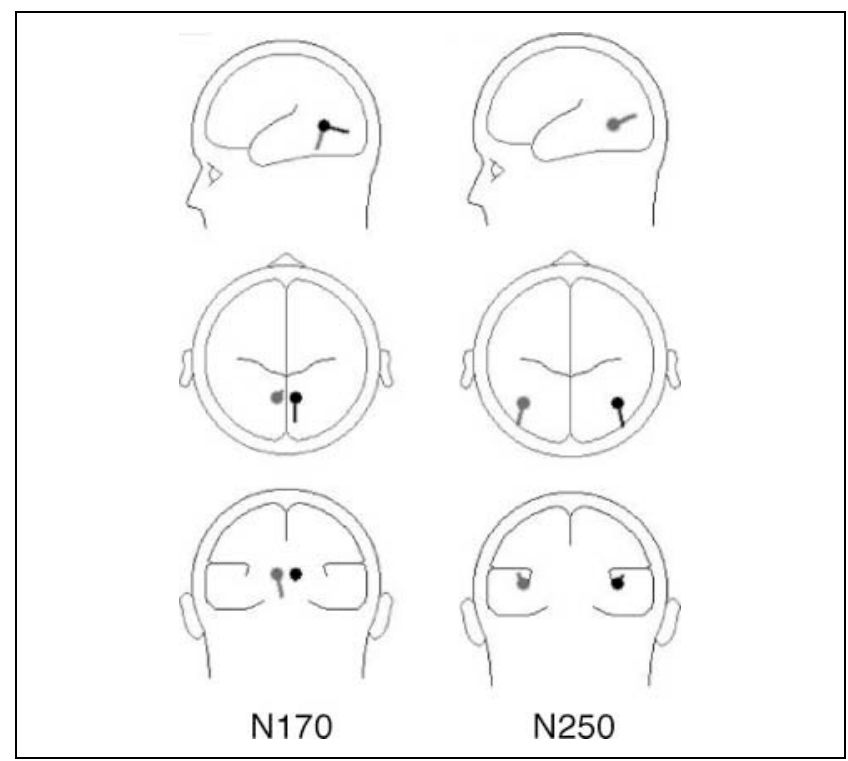

Figure 7. Source modeling. Estimates of source localization for the posttest subordinate-level condition for each component. ence with objects in their domain of expertise rather than their subordinate-level knowledge of these objects (Gauthier et al., 2003; Rossion, Curran, et al., 2002; Tanaka \& Curran, 2001). These results are also consistent with the widely reported face N170 effect (Itier \& Taylor, 2004b; Carmel \& Bentin, 2002; Sagiv \& Bentin, 2001; Rossion et al., 2000; Bentin, Allison, Puce, Perez, \& McCarthy, 1996) in which faces have been shown to elicit a greater N170 relative to other nonface objects (e.g., cars, houses, chairs), but is not sensitive to the relative familiarity of any particular face (Bentin \& Deouell, 2000; Eimer, 2000). Presumably, from birth, humans are exposed to faces more than any other object category, and the years of accumulated experience are manifested in a greater N170 to faces. However, the posttraining N170 in the current study was not restricted to the specific items used in training because this effect extended to untrained exemplars of trained species. Thus, the N170 effect is not limited to the specific episodic experiences, but generalizes to the broader object categories of the perceptual experience.

Whereas the N170 indexed general effects of basiclevel category experience, the N250 component was only sensitive to training at the subordinate level. Specifically, a greater N250 deflection was elicited by participants trained with owls and wading birds at the subordinate level compared to the basic level. Consistent with subordinate-level matching task accuracy, the N250 training effects generalized to new exemplars of trained species and new species within the subordinatelevel family. These results are compatible with recent investigations demonstrating an N250r component to the 
repetition of familiar relative to unfamiliar faces (Tanaka et al., 2006; Schweinberger et al., 2002, 2004). However, the current findings differ from previous N250 experiments in several ways. First, the present study demonstrates that the N250 is not only specific to faces, but also extends to the recognition of nonface objects. Second, our experiment highlights the importance of subordinate-level learning in the amplification of the N250 and is differentiated from the N170 effect that is produced as a consequence of both basic-level and subordinate-level category learning. Finally, whereas previous N250 reports suggest that this component depends on the immediate repetition of familiar stimuli, the current study shows that modulation of the N250 can be demonstrated under conditions over retention intervals lasting several days.

Differences between basic- and subordinate-level object recognition are proposed by some to differ in qualitative ways (Nguyen \& Cottrell, 2005; Collin \& McMullen, 2005; Murphy \& Brownell, 1985; Tversky \& Hemenway, 1984) and by others in only quantitative ways (Riesenhuber \& Poggio, 2002). The present investigation localized the N170 to medial regions of the occipital lobes (lingual gyrus) and the N250 to more lateral regions of the temporal lobes (between the subgyral and inferior temporal gyral regions). Although one must exercise caution when interpreting localized sources using ERPs, here we place less emphasis on the identity of the localized regions than on the simple fact that the N170 and N250 localized to different locations, implying that the present results involve distinct, but likely interactive, brain mechanisms. The regions identified in the present investigation differ slightly from regions previously localized for faces (Schweinberger et al., 2004). Schweinberger et al. (2004) localized the N170 to the lateral occipital face area and the N250 to the fusiform gyrus.

Consistent with the source modeling conducted in the present experiment, previous reports have localized the N170 and N250 to separate brain regions (Schweinberger et al., 2002, 2004). These reports, combined with the present investigation, suggest different neural generators for these two components and are thereby consistent with models positing separate underlying mechanisms related to the learning of basicversus subordinate-level information (e.g., Nguyen \& Cottrell, 2005; Murphy \& Brownell, 1985; Tversky \& Hemenway, 1984). However, given the limitations of source analysis, the results of the present study may also be consistent with a single-system model for subordinate- and basic-level processing (e.g., Riesenhuber \& Poggio, 2000, 2002). Riesenhuber and Poggio (2000, 2002) suggest that basic- and subordinate-level recognition are quantitatively, but not qualitatively, different. In this view, the more fine-grained discrimination required for subordinate-level processing would necessitate the accumulation of more perceptual information compared with that required for basic-level processing. Consistent with the results of the present investigation, this increase in amount of perceptual information needed would lead to a longer latency response for subordinate- compared to basic-level processing. This would suggest that the increased N250 to subordinate- compared to basic-level-trained birds could simply be due to the increased difficulty in subordinate-level discrimination of birds trained at the basic level posed to participants. This interpretation does not require separate systems for basic- and subordinate-level recognition. Future research combining both computational modeling and electrophysiology may be able to more clearly delineate the difference between basic- and subordinatelevel object processing.

The present electrophysiological findings further explain behavioral data (Tanaka et al., 2005) suggesting that there is a shift in categorical entry-level processing from the basic to subordinate levels with increases in perceptual expertise. The ERP results suggest that, similar to general object recognition, perceptual analysis of objects of expertise proceeds from the basic to subordinate levels of analysis. Moreover, the coarse shape information characterized by basic-level categorization occurs earlier in visual processing than the more fine grained visual information required for subordinate-level categorization, regardless of level of expertise. Based on these findings we can conclude that perceptual expertise not only includes increased subordinate-level discrimination of objects within a category of expertise, but is also associated with enhanced basic-level processing. Thus, perceptual expertise might depend on two interacting mechanisms including (1) increases in basic-level shape information (indexed by the N170) and (2) increases in more fine grained subordinate-level discrimination (indexed by the N250). However, it is currently unclear whether there are functionally parallel mechanisms for basic- and subordinate-level processing (e.g., Nguyen \& Cottrell, 2005) or whether basic-level processing simply proceeds and then feeds into subordinatelevel processing (e.g., Jolicoeur et al., 1984).

Our interpretation of the present results is generally consistent with other views of the N170 and N250 within the specific domain of face processing. The face N170 has been related to structural differentiation of faces from other categories of objects (Schweinberger \& Burton, 2003; Sagiv \& Bentin, 2001; Bentin \& Deouell, 2000; Eimer, 2000). The face N250, on the other hand, has been related to the ability to identify individual faces (Schweinberger et al., 2002, 2004). Thus, just as others have suggested that the N170 and N250 may index separate processes in face recognition, the present results suggest that these components may reflect more domain-general learning/categorization mechanisms. Like studies of real-world expertise, studies of face recognition are limited in their ability to measure the processes involved in the acquisition of categorical 
knowledge. The present study sheds light on these processes by measuring electrophysiological changes that result from category learning under controlled conditions.

\section{Acknowledgments}

This research was supported by the James S. McDonnell Foundation, a grant to T. C. from the National Institute of Mental Health (MH64812), and a grant to J.T. from Natural Sciences and Engineering Research Council of Canada.

The authors thank members of the Perceptual Expertise Network for relevant discussion, and D. Chase, R. Danson, C. DeBuse, D. Germain, J. Hancock, J. Knellinger, B. Malcolm, R. Praw, E. Walsky, B. Woroch, and B. Young for technical and research assistance.

Reprint requests should be sent to Lisa S. Scott, Department of Psychology, University of Massachusetts, 413 Tobin Hall/135 Hicks Way, Amherst, MA 01003 or Tim Curran, Department of Psychology, University of Colorado, 345 UCB, Boulder, CO 80309-0345, or via e-mail: lscott@psych.umass.edu or tim. curran@colorado.edu.

\section{REFERENCES}

Bentin, S., Allison, T., Puce, A., Perez, E., \& McCarthy, G. (1996). Electrophysiological studies of face perception in humans. Journal of Cognitive Neuroscience, 8 , 551-565.

Bentin, S., \& Deouell, L. Y. (2000). Structural encoding and identification in face processing: ERP evidence for separate mechanisms. Cognitive Neuropsychology, 17, 35-54.

Biederman, I. (1985). Human image understanding: Recent research and theory. Computer Vision, Graphics, and Image Processing, 32, 29-73.

Biederman, I., \& Gerhardstein, P. C. (1993). Recognizing depth-rotated objects: Evidence and conditions for three-dimensional view point invariance. Journal of Experimental Psychology: Human Perception and Performance, 18, 121-133.

Busey, T. A., \& Vanderkolk, J. R. (2005). Behavioral and electrophysiological evidence for configural processing in fingerprint experts. Vision Research, 45, 431-448.

Carmel, D., \& Bentin, S. (2002). Domain specificity versus expertise: Factors influencing distinct processing of faces. Cognition, 83, 1-29.

Collin, C. A., \& McMullen, P. (2005). Subordinate-level categorization relies on high spatial frequencies to a greater degree than basic-level categorization. Perception E Psychophysics, 67, 354-364.

Curran, T., Tanaka, J. W., \& Weiskopf, D. M. (2002). An electrophysiological comparison of visual categorization and recognition memory. Cognitive, Affective, and Behavioral Neuroscience, 2, 1-18.

Eimer, M. (2000). The face-specific N170 component reflects late stages in the structural encoding of faces. NeuroReport, 11, 2319-2324.

Gauthier, I., Curran, T., Curby, K. M., \& Collins, D. (2003). Perceptual interference supports a non-modular account of face processing. Nature Neuroscience, 6, 428-432.

Gauthier, I., Tarr, M. J., Moylan, J., Anderson, A. W., Skudlarski, P., \& Gore, J. C. (2002). Does subordinate-level categorization engage the functionally-defined face area? Cognitive Neuropsychology, 17, 143-163.
Grill-Spector, K., \& Kanwisher, N. (2005). Visual recognition: As soon as you know it is there, you know what it is. Psychological Science, 16, 152-160.

Itier, R. J., \& Taylor, M. J. (2004a). Effects of repetition learning on upright, inverted and contrast-reversed face processing using ERPs. Neuroimage, 21, 1518-1532.

Itier, R. J., \& Taylor, M. J. (2004b). N170 or N1? Spatiotemporal differences between object and face processing using ERPs. Cerebral Cortex, 14, 132-142.

Jolicoeur, P. (1990). Identification of disoriented objects: A dual-systems theory. Mind E Language, 5, 387-410.

Jolicoeur, P., Gluck, M., \& Kosslyn, S. M. (1984). Pictures and names: Making the connection. Cognitive Psychology, 16, 243-275.

Kimchi, R. (1992). Primacy of wholistic processing and global/local paradigm: A critical review. Psychological Bulletin, 112, 24-38.

Logothetis, N. K., \& Sheinberg, D. L. (1996). Visual object recognition. Annual Review of Neuroscience, 19, 577-621.

Murphy, G. L., \& Brownell, H. H. (1985). Category differentiation in object recognition: Typicality constraints on the basic category advantage. Journal of Experimental Psychology: Learning, Memory, and Cognition, 11, 70-84.

Nguyen, N., \& Cottrell, G. W. (2005). Owls and wading birds: Generalization gradients in expertise. In Proceedings of the 27th Annual Cognitive Science Conference, La Stresa, Italy (pp. 1636-1641). Mahwah, NJ: Erlbaum.

Riesenhuber, M., \& Poggio, T. (2000). Models of object recognition. Nature Neuroscience, 3, 1199-1204.

Riesenhuber, M., \& Poggio, T. (2002). Neural mechanisms of object recognition. Current Opinion in Neurobiology, 12, 162-168.

Rossion, B., Curran, T., \& Gauthier, I. (2002). A defense of the subordinate-level expertise account for the N170 component. Cognition, 85, 189-196.

Rossion, B., Dricot, L., Devolder, A., Bodart, J. M., Crommelinck, M., De Gelder, B., \& Zoontjes, R. (2000). Hemispheric asymmetries for whole-based and part-based face processing in the human fusiform gyrus. Journal of Cognitive Neuroscience, 12, 793-802.

Rossion, B., Gauthier, I., Goffaux, V., Tarr, M. J., \& Crommelinck, M. (2002). Expertise training with novel objects leads to left-lateralized facelike electrophysiological responses. Psychological Science, 13, 250-257.

Sagiv, N., \& Bentin, S. (2001). Structural encoding of human and schematic faces: Holistic and part-based processes. Journal of Cognitive Neuroscience, 13, 937-951.

Scherg, M., \& Berg, P. (1996). New concepts of brain source imaging and localization. Electroencephalography and Clinical Neurophysiology Supplement, 46, 127-137.

Schweinberger, S. R., \& Burton, A. M. (2003). Covert recognition and the neural system for face processing. Cortex, 39, 9-30.

Schweinberger, S. R., Huddy, V., \& Burton, M. (2004). N250r: A face-selective brain response to stimulus repetitions. NeuroReport, 15, 1501-1505.

Schweinberger, S. R., Pickering, E. C., Burton, M., \& Kaufmann, J. (2002). Human brain potential correlates of repetition priming in face and name recognition. Neuropsychologia, 40, 2057-2073.

Schyns, P. G., \& Oliva, A. (1994). From blobs to boundary edge: Evidence for time- and spatial-scale-dependent scene recognition. Psychological Science, 5, 195-200.

Srinivasan, R., Nunez, P. L., Silberstein, R. B., Tucker, D. M., \& Cadusch, P. J. (1996). Spatial sampling and filtering 
of EEG with spline-Laplacians to estimate cortical potentials. Brain Topography, 8, 355-366.

Tanaka, J. W. (2001). The entry point of face recognition: Evidence for face expertise. Journal of Experimental Psychology: General, 130, 534-543.

Tanaka, J. W., \& Curran, T. (2001). A neural basis for expert object recognition. Psychological Science, 12, 43-47.

Tanaka, J. W., Curran, T., Porterfield, A. L., \& Collins, D. (2006). Activation of preexisting and acquired face representations: The N250 event-related potential as an index of face familiarity. Journal of Cognitive Neuroscience, 18, 1488-1497.

Tanaka, J. W., Curran, T., \& Sheinberg, D. L. (2005). The training and transfer of real-world, perceptual expertise. Psychological Science, 16, 145-151.

Tanaka, J. W., \& Taylor, M. (1991). Object categories and expertise: Is the basic level in the eye of the beholder? Cognitive Psychology, 23, 457-482.

Tucker, D. M. (1993). Spatial sampling of head electrical fields: The geodesic sensor net. Electroencephalography and Clinical Neurophysiology, 87, 154-163.

Talairach, J., \& Tournoux, P. (1988). Co-planar stereotaxic atlas of the buman brain. Stuttgart: Thieme.

Tversky, B., \& Hemenway, K. (1984). Objects, parts, and categories. Journal of Experimental Psychology: General, 113, 169-197. 